\title{
Association between Adipokines and Biomarkers of Alzheimer's Disease: A Cross-Sectional Study
}

\author{
Liliana Letra $^{\mathrm{a}, *}$, Paulo Matafome ${ }^{\mathrm{a}}$, Tiago Rodrigues ${ }^{\mathrm{a}}$, Diana Duro ${ }^{\mathrm{b}, \mathrm{c}}$, Raquel Lemos ${ }^{\mathrm{b}}$, Inês \\ Baldeiras $^{\mathrm{b}, \mathrm{c}, \mathrm{d}}$, Miguel Patrício ${ }^{\mathrm{c}}$, Miguel Castelo-Branco ${ }^{\mathrm{c}, \mathrm{e}}$, Gina Caetano ${ }^{\mathrm{c}, \mathrm{e}}$, Raquel Seiça ${ }^{\mathrm{a}}$ \\ and Isabel Santana ${ }^{\mathrm{b}, \mathrm{c}, \mathrm{d}}$ \\ ${ }^{a}$ Institute of Physiology and Coimbra Institute for Clinical and Biomedical Researh (iCBR), \\ Faculty of Medicine, University of Coimbra, Portugal \\ ${ }^{\mathrm{b}}$ Department of Neurology, Centro Hospitalar e Universitário de Coimbra, Portugal \\ ${ }^{\mathrm{c}}$ Faculty of Medicine, University of Coimbra, Portugal \\ ${ }^{\mathrm{d}}$ Center for Neuroscience and Cell Biology $(C N C)$, University of Coimbra, Portugal \\ ${ }^{\mathrm{e}}$ Institute of Nuclear Sciences Applied to Health (ICNAS), University of Coimbra, Portugal
}

Accepted 19 November 2018

\begin{abstract}
.
Background: Adipose tissue dysfunction has been implicated in the pathophysiology of Alzheimer's disease. However, the involvement of adipokines, particularly adiponectin, remains unclear.

Objective: To compare serum and cerebrospinal fluid (CSF) levels of adiponectin, leptin and leptin-to-adiponectin ratio in patients within the spectrum of Alzheimer's disease and evaluate their relationship with classical biomarkers and their value as markers of progression.

Methods: Amnestic mild cognitive impairment (MCI, $n=71$ ) and Alzheimer's dementia (AD, $n=53)$ subjects were consecutively recruited for serum and CSF adiponectin and leptin determination using an analytically validated commercial enzyme-linked immunosorbent assay (ELISA). Correlations were explored using adjusted Spearman's correlation coefficients. A logistic regression model and ROC analysis were performed to evaluate the staging predictive value of adipokines. Results: Serum adiponectin was 33\% higher in AD when compared to MCI patients. Adiponectin CSF levels, similar in both groups, were positively correlated with $\mathrm{A} \beta_{42}$ and cognitive function, though only in women. The area under the ROC curve was 0.673 (95\%CI:0.57-0.78) for serum adiponectin as predictor of dementia stage and the cut-off $10.85 \mu \mathrm{g} / \mathrm{ml} \mathrm{maximized}$ the sum of specificity $(87 \%)$ and sensitivity $(44 \%)$.

Conclusion: Although longitudinal studies are required, we hypothesize that higher serum adiponectin in AD patients constitutes a strategy to compensate possible central signaling defects. In addition, adiponectin might be specifically assigned to neuroprotective functions in women and eventually involved in the female-biased incidence of Alzheimer's disease.
\end{abstract}

Keywords: Adipokines, adiponectin, adipose tissue, Alzheimer's disease, biomarkers, cerebrospinal fluid, leptin

\footnotetext{
${ }^{*}$ Correspondence to: Liliana Letra, Institute of Physiology and Coimbra Institute for Clinical and Biomedical Research (iCBR), Faculty of Medicine, University of Coimbra, Azinhaga de Santa Comba, 3000-548 Coimbra, Portugal. Tel.: +00351 91935895; Email: 1rletra@fmed.uc.pt.
}

\section{INTRODUCTION}

In the last two decades, undeniable epidemiological evidence has emerged indicating middle-age obesity as a risk factor for Alzheimer's disease [1]. Nevertheless, the pathophysiological mechanisms 
implicated are only scarcely disclosed. It is already recognized that adipose tissue dysfunction contributes to cerebrovascular disease which in turn promotes and may even precede neurodegeneration [2]. But on the other hand, several studies have highlighted vascular-independent contributions of obesity to Alzheimer's disease, particularly through adipokine-mediated effects on the central nervous system (CNS) [3, 4]. It is unclear though to what extent adipokines may influence cerebral structure and function and which mechanisms are involved. Adipokines are proteins mainly secreted by the white adipose tissue that are involved in important physiological functions such as regulation of appetite and energy expenditure, glucose homeostasis, inflammation, and endothelial function [5-7]. First described in the $1990 \mathrm{~s}$, adipokines have already proved their clinical relevance as biomarkers of adipose tissue dysfunction and its related disorders [8, 9]. More recently, adipokine's neuroprotective actions and their specific beneficial effects on memory have also been reported. Leptin is able to enhance hippocampal long-term potentiation and synaptic plasticity, as well as to promote non-amyloidogenic processing of amyloid- $\beta$ protein precursor and prevent tau hyperphosphorylation [10]. On the contrary, a beneficial role of adiponectin in Alzheimer's disease natural history is still controversial, in spite of its recognized insulin-sensitizing, anti-inflammatory, anti-apoptotic, and anti-atherosclerotic properties [11] as well as recent reports of its capacity to promote dendritic growth, arborization, and spinogenesis in mature dentate gyrus neurons [12]. Besides, there is no published study addressing leptin-to-adiponectin ratio (LAR) alterations in this disease or its association with biomarkers. Although serum LAR is recognized as a more reliable marker of adipose tissue dysfunction and of its metabolic effects than adipokine serum levels separately [13, 14], there is no evidence of its clinical relevance when measured in the cerebrospinal fluid (CSF). Therefore, this study aims to explore the role of the two most abundant circulating adipokines and their ratio in adipose tissue-brain crosstalks in this disease context. It evaluates serum and CSF levels of adiponectin, leptin, and LAR in patients with amnestic mild cognitive impairment (MCI or prodromal phase) and mildto-moderate Alzheimer's dementia (AD). It further explores correlations between adipokine levels with CSF and imaging biomarkers of the disease, as well as gender differences that may contribute to the femalebiased incidence of Alzheimer's disease.

\section{MATERIALS AND METHODS}

\section{Participants and clinical evaluation}

The study was conducted in a group of 124 subjects consecutively recruited at the Dementia outpatient Clinic of Centro Hospitalar e Universitário de Coimbra (CHUC). They were extensively characterized until a final probable diagnosis of an Alzheimer's disease spectrum disorder. We included 53 patients with a probable diagnosis of dementia due to Alzheimer's disease (mild and moderate stages) that we further refer as $\mathrm{AD}$, and 71 patients with the diagnosis of amnestic MCI. A group of healthy or asymptomatic/pre-clinical individuals was not considered since performing a lumbar puncture in these individuals would not be ethically acceptable, as is also the case of patients in severe dementia stages whose clinical benefit of CSF biomarker analysis is minimal. At baseline, patients and an informant underwent a structured clinical interview conducted by a neurologist. It included a detailed description of complaints, with special focus on time course and leading symptoms. Handedness, past medical and family history as well as drug history were also registered. A complete neurological examination was performed to rule out non-Alzheimer's disease causes of cognitive decline and weight, height, waist and hip circumferences were measured according to the recommendations of World Health Organization Expert Consultation report [15]. Body mass index (BMI) was calculated using the following formula: $\mathrm{BMI}=$ body weight (kilograms)/height ${ }^{2}$ (meters). Patients also underwent biochemical evaluation and brain imaging (computed tomography and/or MRI) to exclude other forms of dementia and most of them were also studied with CSF biomarkers and APOE genotyping. Probable Alzheimer's dementia was diagnosed according to the Diagnostic and Statistical Manual of Mental Disorders criteria [16], the National Institute of Neurological and Communicative Disorders and Stroke-Alzheimer's Disease and Related Disorders (NINCDS-ADRDA) [17] and more recently to National Institute on Aging and the Alzheimer's Association (NIAAS) revised criteria [18]. MCI patients were selected according to Petersen's criteria [19] and the likelihood of underlying Alzheimer's disease pathophysiology was assumed based on CSF biomarker profile and/or neuropsychological profile consistent with amnestic syndrome of the hippocampal type. Clinical Dementia Rating (CDR) [20, 21] of $0.5,1$, and 2 as well as neuropsychological 
battery tests scores (described below) were indicative of MCI, mild and moderate dementia, respectively. All participants or respective caregivers gave informed consent to participate and all the procedures were conducted in accordance with the ethical review board of the Centro Hospitalar e Universitário de Coimbra (CHUC).

\section{Neuropsychological evaluation}

The neuropsychological protocol included a battery of tests composed by the Mini-Mental State Examination (MMSE) Portuguese version [22, 23], the Montreal Cognitive Assessment (MoCA) [24, 25] Portuguese version, and the Alzheimer Disease Assessment Scale-Cognitive (ADAS-Cog) Portuguese version [26, 27]. Assessment was performed by experienced neuropsychologists who also administered the Portuguese versions of the CDR for global staging and the Geriatric Depression Scale (GDS-30) [28, 29] to exclude major depression.

\section{Peripheral blood and CSF collection and determinations}

Blood samples were collected for general metabolic and adipokine determinations as well as for APOE genotyping. The homeostatic model assessment of insulin-resistance index (HOMA-IR) was calculated as [(If)*(Gf)]/22.5, where (If) is the fasting insulin level (mU/ml) and (Gf) is the fasting glucose level (mmol/L). Serum cholesterol (total and highdensity lipoprotein - HDL), triglyceride and fasting glycemia were determined using commercial kits (Olympus-Diagnóstica, Produtos de Diagnóstico SA, Portugal). Low-density lipoprotein (LDL) cholesterol was calculated according to the Friedewald equation [30]. Leptin, adiponectin, and insulin were determined using Human Adiponectin and Leptin ELISA Kit (DuoSet, R\&D Systems) and Human Insulin ELISA kit (Mercodia, Sweden), respectively. APOE genotype was determined by PCR-restriction fragment length polymorphisms (RFLP) assay after DNA isolation from whole EDTA-blood using a commercial kit (Roche Diagnostics GmbH, Manheim, Germany). Regarding CSF, pre-analytical and analytical procedures were done in accordance with previously proposed protocols [31]. CSF A $\beta_{42}$, total tau (t-tau), and phosphorylated tau (p-tau 181 ) were measured separately by commercially available sandwich ELISA kits (Innotest, Innogenetics, Ghent, Belgium), as previously described [32]. Leptin and adiponectin CSF concentrations were also measured by sandwich ELISA (DuoSet, R\&D Systems).

\section{MRI data collection}

A sub-group of participants (36 MCI, 31 AD) underwent MRI on a 3T Siemens Magnetom Trio scanner (Erlangen, Germany), using a 12channel birdcage head coil. High-resolution 3D T1- weighted magnetization-prepared rapid gradient echo (MP-RAGE) scans were collected per participant, with parameters defined on the basis of guidelines from the Alzheimer's Disease Network Initiative [33]. Structural MRI scans were processed by the FreeSurfer 5.0.0 software package (http://surfer.nmr.mgh.harvard.edu ) using fully automated methods which are elsewhere described [34, 35]. Briefly, for each participant, cortical thickness was estimated at each point of the cortical mantle by finding the shortest distance from the white matter surface to the grey matter surface (and vice-versa) and averaging those two values. The neocortex was parcellated into 32 gyral-based regions-of-interest (ROI) in each hemisphere, and in addition non-neocortical ROIs, such as hippocampus, were defined on the basis of automated procedures. Total hippocampal volume was calculated by summing up right and left hippocampal volumes. For each participant, the accuracy of the determination of the grey and white matter surfaces and of each individual ROI was carefully inspected by a trained neuroradiologist.

\section{Statistical analysis}

A power analysis was performed in Gpower 3.1.9.2, concerning the comparison between serum adiponectin levels measured for $\mathrm{AD}$ and $\mathrm{MCI}$ groups. The resulting power was $85.6 \%$. Each quantitative variable was assessed for normality resorting to Shapiro-Wilk tests and graphical analysis. When found to be normally distributed, they were described by their mean and standard deviation. Otherwise, the median, 25th and 75th percentiles were used instead. Categorical variables were described in terms of absolute and relative frequencies. Comparisons of quantitative variables between categories were performed with t-Student tests, whenever the normality assumptions held, and with Mann-Whitney U tests otherwise. The associations between pairs of categorical variables were assessed with chi-square tests, whereas correlations between pairs of quantitative variables were assessed by computing Spearman's 
correlation coefficients. Partial Spearman correlation coefficients were also computed to account for the influence of covariables. The analysis was performed in $\mathrm{R}$ version 3.3.2 and the plots in IBM SPSS Statistics 24 . The level of significance adopted was 0.05 .

\section{RESULTS}

\section{Baseline characteristics and adipokine profile of} the study participants

Demographic, clinical, and genetic characteristics of the study participants are listed in Table 1. There were no significant differences between MCI and $\mathrm{AD}$ patients regarding age, gender, education, and percentage of APOE\&4 carriers. Anthropometric measurements as well as typical vascular risk factors and co-morbid metabolic diseases were also similar in both groups. As expected, MMSE and MoCA mean-scores were lower and ADAS-Cog scores were higher in the $\mathrm{AD}$ group $(p<0.001)$. Additional serum and CSF biochemical and biomarker data are presented in Tables 2 and 3, respectively. A significant difference in serum adiponectin levels between $\mathrm{AD}$ and $\mathrm{MCI}$ patients was observed, with the former attaining 33\% higher levels (Fig. 1). On the contrary, serum leptin and LAR were equivalent in both groups as well as CSF levels of both adipokines and LAR.

\section{Serum adiponectin as a staging biomarker of Alzheimer's disease}

A logistic regression model was performed where the patient status (MCI or $\mathrm{AD}$ ) was taken as the dependent variable and the following independent variables were considered: serum and CSF leptin, serum and CSF adiponectin, and HOMA-IR. This was done after verifying that the strengths of the correlations between the independent variables would not lead to concerns with co-linearity: in particular the correlations between serum and CSF adiponectin $(r=0.532, p=0.044)$ and between serum and CSF leptin $(r=0.532, p=0.044)$, which were both moderate. Out of the independent variables, only serum adiponectin was found to significantly contribute to the model. Applying backward elimination, only this variable remained significant, which achieved statistical significance: $\chi^{2}(1)=9.547, \quad p=0.002$, Nagelkerke $R^{2}=0.114$. Finally, taking serum and

Table 1

Demographic, clinical, and genetic data of study participants, according to baseline diagnosis

\begin{tabular}{|c|c|c|c|}
\hline & $\begin{array}{c}\text { MCI } \\
n=71\end{array}$ & $\begin{array}{c}\mathrm{AD} \\
n=53\end{array}$ & $p$ \\
\hline Age (y) & $73(67 ; 76)$ & $75(68 ; 80)$ & 0.060 \\
\hline Age onset (y) & $67.33 \pm 8.30$ & $68.53 \pm 9.08$ & 0.507 \\
\hline Female gender $\mathrm{n}(\%)$ & $48(67.6 \%)$ & $37(69.8 \%)$ & 0.794 \\
\hline Education (y) & $4(4 ; 6.75)$ & $4(4 ; 9)$ & 0.620 \\
\hline ApoE $\varepsilon 4$ carrier $\mathrm{n}(\%)$ & $41(61.2 \%)$ & $23(45.1 \%)$ & 0.082 \\
\hline BMI & $26.36 \pm 3.71$ & $25.24 \pm 4.22$ & 0.143 \\
\hline $\mathrm{WC}(\mathrm{cm})$ & $96.39 \pm 10.70$ & $92.45 \pm 11.93$ & 0.083 \\
\hline $\mathrm{HC}(\mathrm{cm})$ & $102(96.25 ; 107)$ & $100(96 ; 103)$ & 0.202 \\
\hline WHR & $0.94(0.89 ; 1.00)$ & $0.91(0.87 ; 0.96)$ & 0.074 \\
\hline Hypertension n (\%) & $38(54.3 \%)$ & $19(38 \%)$ & 0.078 \\
\hline Type 2 DM n (\%) & $13(18.6 \%)$ & $13(26 \%)$ & 0.330 \\
\hline Dyslipidemia n (\%) & $45(64.3 \%)$ & $24(48 \%)$ & 0.075 \\
\hline Heart Failure n (\%) & $8(11.4 \%)$ & $2(4 \%)$ & 0.191 \\
\hline $\operatorname{MetS}^{1} \mathrm{n}(\%)$ & $27(38.6 \%)$ & $13(24.1 \%)$ & 0.242 \\
\hline MMSE & $27(25 ; 29)$ & $19(15.75 ; 23.25)$ & $<0.001$ \\
\hline ADAS-Cog & $11(7 ; 13)$ & $21(16 ; 27.25)$ & $<0.001$ \\
\hline $\mathrm{MoCA}$ & $19.04 \pm 4.94$ & $10.38 \pm 4.89$ & $<0.001$ \\
\hline
\end{tabular}

Data presented as mean \pm standard deviation, median (1st quartile, 3rd quartile) or as number (percentage) of patients, as applicable. The $p$-values $(p)$ included in the table were obtained with independent samples t-Student tests or Mann-Whitney U tests for quantitative variables and with chi-square tests for qualitative variables. ${ }^{1}$ MetS defined according to the International Diabetes Federation (IDF). AD, Alzheimer's disease; ADAS-Cog, Alzheimer's Disease Assessment Scale-Cognitive subscale; ApoE \&4, Apolipoprotein E allele 4; BMI, body mass index; DM, diabetes mellitus; HC, hip circumference; MCI, mild cognitive impairment; MetS, metabolic syndrome; MMSE, Mini-Mental State Examination; MoCA, Montreal Cognitive Assessment; WC, waist circumference; WHR; waist-to-hip ratio. 
Table 2

Serum biochemical data of study participants, according to baseline diagnosis

\begin{tabular}{lccc}
\hline & MCI & AD & $p$ \\
& $n=71$ & $n=53$ & \\
\hline Fasting glycemia $(\mathrm{mg} / \mathrm{dl})$ & $89(83 ; 97.25)$ & $92(86 ; 105)$ & 0.140 \\
Fasting insulinemia (mU/L) & $4.92(3.68 ; 5.86)$ & $5.10(4.21 ; 7.02)$ & 0.252 \\
HOMA-IR & $1.11(0.84 ; 1.42)$ & $1.23(0.92 ; 1.77)$ & 0.196 \\
Total cholesterol $(\mathrm{mg} / \mathrm{dl})$ & $196(176 ; 238)$ & $207(187 ; 233)$ & 0.354 \\
LDL-cholesterol $(\mathrm{mg} / \mathrm{dl})$ & $121(99.2 ; 161.4)$ & $137(115.8 ; 155.2)$ & 0.122 \\
HDL-cholesterol $(\mathrm{mg} / \mathrm{dl})$ & $53.08 \pm 12.70$ & $54.68 \pm 11.81$ & 0.524 \\
Triglycerides $(\mathrm{mg} / \mathrm{dl})$ & $109(80 ; 140)$ & $93.50(73 ; 130.75)$ & 0.235 \\
CRP $(\mathrm{mg} / \mathrm{dl})$ & $0.23(0.11 ; 0.39)$ & $0.20(0.07 ; 0.38)$ & 0.278 \\
Adiponectin $(\mu \mathrm{g} / \mathrm{ml})$ & $6.52(3.86 ; 9.44)$ & $9.80(6.03 ; 14.30)$ & $\mathbf{0 . 0 0 2}$ \\
Leptin $(\mathrm{ng} / \mathrm{ml})$ & $9.97(6.44 ; 21.79)$ & $10.12(6.64 ; 19.40)$ & 0.698 \\
LAR & $1.87(1.01 ; 4.27)$ & $1.44(0.71 ; 3.03)$ & 0.142 \\
\hline
\end{tabular}

Data presented as mean \pm standard deviation or median (1st quartile; 3 rd quartile), as applicable. The $p$-values $(p)$ included in the table were obtained with independent samples t-Student tests or Mann-Whitney U tests, as applicable. AD, Alzheimer's disease; CRP, C reactive protein; HDL, high-density cholesterol; HOMA-IR,: Homeostasis Model Assessment-Insulin Resistance; LAR, leptin to adiponectin ratio; LDL, low-density cholesterol; MCI, mild cognitive impairment.

Table 3

Cerebrospinal fluid biomarker and biochemical data of study participants, according to baseline diagnosis

\begin{tabular}{lccccc}
\hline & MCI & $n$ & AD & $n$ & $p$ \\
\hline $\mathrm{A} \beta_{42}(\mathrm{pg} / \mathrm{mL})$ & $703.99 \pm 340.18$ & 37 & $425.58 \pm 162.31$ & 32 & $<\mathbf{0 . 0 0 1}$ \\
t-tau $(\mathrm{pg} / \mathrm{mL})$ & $382.9(228.2 ; 520.7)$ & 37 & $615.75(449.33 ; 817.68)$ & 32 & $<\mathbf{0 . 0 0 1}$ \\
p-tau $(\mathrm{pg} / \mathrm{mL})$ & $46.60(32.7 ; 64.6)$ & 37 & $73(55.075 ; 85.55)$ & 32 & $<\mathbf{0 . 0 0 1}$ \\
t-tau/A $\beta_{42}$ & $0.74(0.31 ; 1.02)$ & 37 & $1.33(1.06 ; 2.50)$ & 32 & $<\mathbf{0 . 0 0 1}$ \\
$\mathrm{A} \beta_{42} / \mathrm{p}$-tau & $12.30(8.67 ; 27.71)$ & 37 & $5.75(4.08 ; 7.47)$ & 32 & $<\mathbf{0 . 0 0 1}$ \\
$\mathrm{ATI}$ & $0.73(0.52 ; 1.29)$ & 37 & $0.41(0.25 ; 0.54)$ & 32 & $<\mathbf{0 . 0 0 1}$ \\
Adiponectin $(\mathrm{ng} / \mathrm{ml})$ & $2.57(1.85 ; 4.26)$ & 34 & $2.93(1.80 ; 4.85)$ & 22 & 0.656 \\
Leptin $(\mathrm{pg} / \mathrm{ml})$ & $163.07(122.19 ; 227.29)$ & 34 & $147.02(98.58 ; 174.38)$ & 22 & 0.135 \\
LAR & $63.63(34.34 ; 121.19)$ & 34 & $41.64(27.28 ; 99.53)$ & 22 & 0.235 \\
\hline
\end{tabular}

Data presented as mean \pm standard deviation or median (1st quartile; 3rd quartile), as applicable. The $p$-values (p) included in the table were obtained with independent samples t-Student tests or Mann-Whitney U tests, as applicable. A $\beta$, amyloid-beta; AD, Alzheimer's disease; ATI, Amyloid-Tau Index; LAR, leptin to adiponectin ratio; $\mathrm{MCI}$, mild cognitive impairment; t-tau, total tau; p-tau, phosphorylated tau.

CSF leptin, serum and CSF adiponectin as a starting point and adding confounding variables to the logistic regression (hypertension, type 2 diabetes mellitus, dyslipidemia, heart failure) results in a statistical significant model, $\chi^{2}(8)=1892, p<0.001$, Nagelkerke $\mathrm{R}^{2}=0.447$. Serum adiponectin's contribution to the model is again statistically significant $(p=0.018)$, with no other variable displaying statistically significant contributions. A ROC analysis was performed to evaluate the predictive value of serum adiponectin, with the $95 \%$ confidence interval for the area under the curve (AUC) being [0.57, 0.78]. The cut-off $10.85 \mu \mathrm{g} / \mathrm{ml}$ maximizes the sum of the specificity and sensitivity, which are $87 \%$ and $44 \%$, respectively (Fig. 2).

\section{Association between adipokines and core Alzheimer's disease biomarkers}

\section{Cerebrospinal fluid biomarkers}

In MCI patients, no correlation was found between the concentration of both adipokines and CSF A $\beta_{42}$, t-tau, and p-tau. In the AD group, out of the three core CSF biomarkers only t-tau was inversely correlated with CSF leptin after adjustments for age, gender, and BMI $(\rho=-0.597, p=0.026)$. Meanwhile, in female patients (MCI and AD), we observed a strong positive correlation between CSF levels adiponectin and $\mathrm{A} \beta_{42}$, which remained significant after age and BMI adjustments $(\rho=0.590, p=0.002)$ (Supplementary Table 1). 

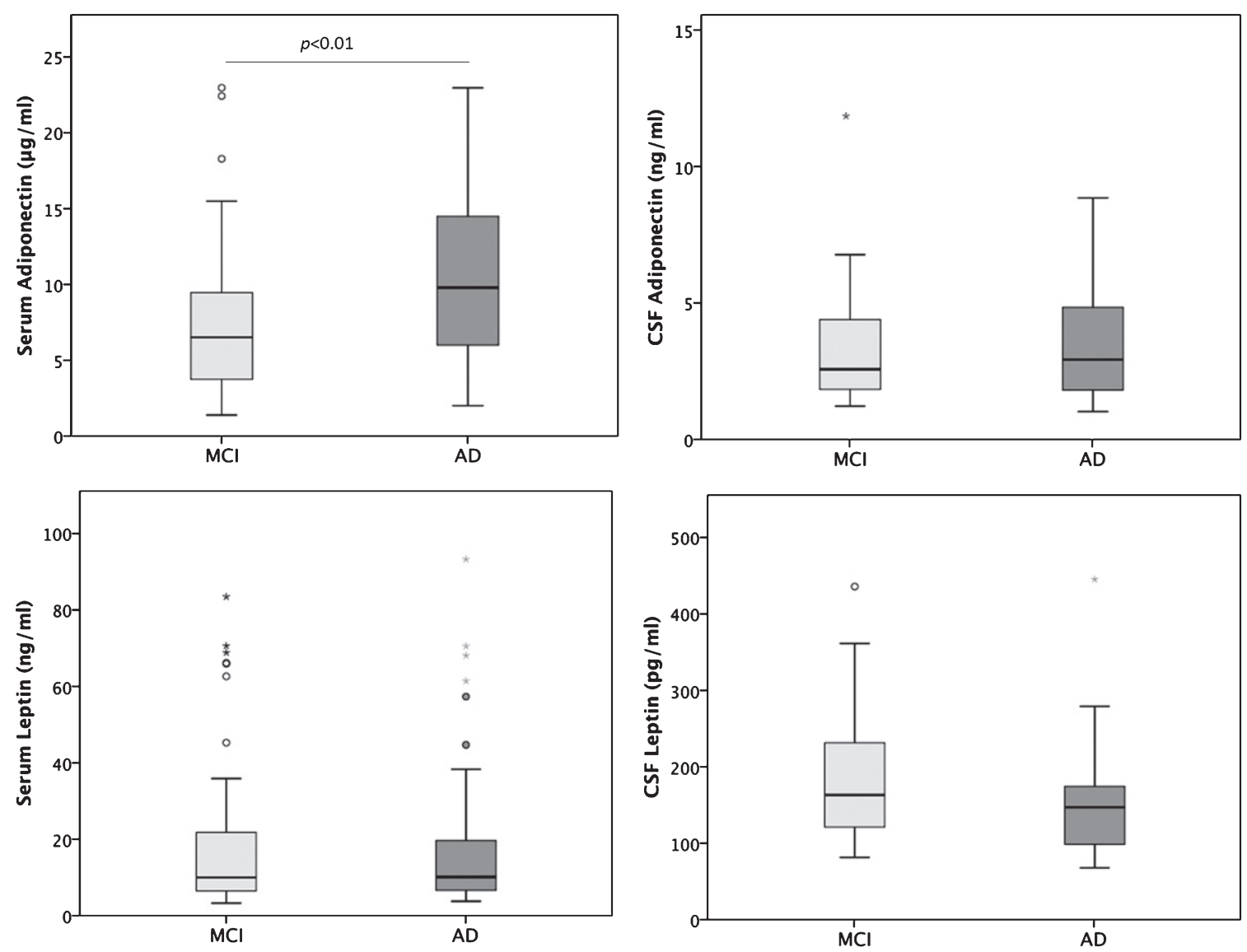

Fig. 1. Serum and CSF concentrations of adiponectin and leptin among MCI and AD subjects. Box and Whisker plots represent median, upper median, lower median and minimum to maximum range of the adipokine concentrations. Only serum adiponectin reached statistically significant difference $(p<0.01)$ between the two groups.

\section{Imaging biomarkers}

No significant correlation was observed between adipokine levels and MRI volumetric measures of medial temporal lobe (MTL) structures in $\mathrm{AD}$ and MCI patients. Furthermore, and based on the fact that women with Alzheimer's disease seem to experience faster hippocampal atrophy [36], we performed the same analysis in this subgroup, but did not observe consistent correlations (Supplementary Table 2).

\section{Association between adipokines and neuropsychological tests}

The total scores of MMSE, MoCA, and ADASCog were not correlated with serum or CSF levels of both adipokines. Only serum leptin demonstrated a tendency to negatively correlate with MoCA and ADAS-Cog scores in the whole cohort after gender,

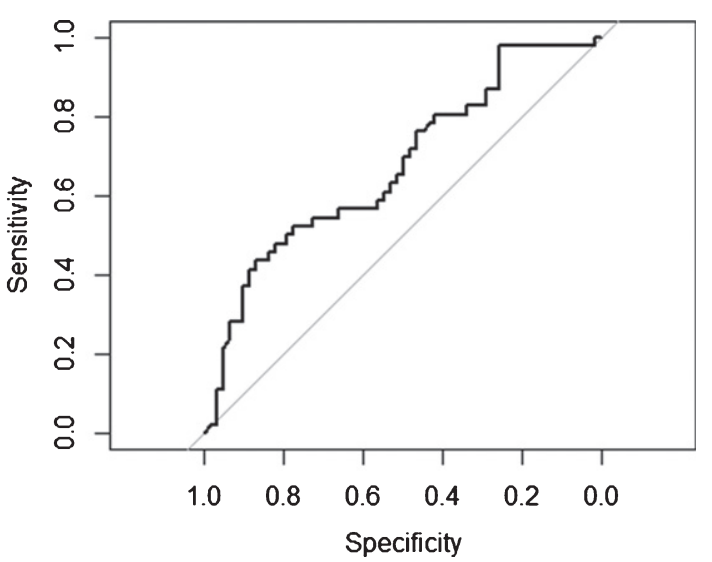

Fig. 2. ROC curve for serum adiponectin as predictor of dementia phase of $\mathrm{AD}$. The reference line represents the ROC curve for a statistical test with no discriminatory ability. The area under the ROC curve was 0.673 (95\%CI: 0.57-0.78) and cut-off level was $10.85 \mu \mathrm{g} / \mathrm{ml}$ (sensitivity $=0.44$; specificity $=0.87$ ). 
age, education, and BMI adjustments. However, in female patients both MoCA and ADAS-Cog were strongly correlated with CSF adiponectin, in the sense that higher concentration was associated with better cognitive function $(\rho=0.611, p=0.046$ and $\rho=-0.548, p=0.028$, respectively). In addition, MoCA scores were inversely correlated with CSF leptin and CSF LAR $(\rho=-0.641, p=0.033$ and $\rho=-0.627, p=0.039$, respectively) (Supplementary Table 3).

\section{DISCUSSION}

The present study investigated the association between adipose tissue and Alzheimer's disease focusing on the alterations in serum and CSF levels of adiponectin and leptin. For that purpose, we considered prodromal (MCI) and mild to moderate dementia phases of the disease. This study also aimed to clarify the epidemiological link between mid-life obesity and late-onset Alzheimer's disease as well as its gender incidence bias. It provides novel biomarkerbased evidence of the relationship between adipokines and $\mathrm{AD}$ pathology and proposes a blood-based staging biomarker.

\section{Adipokine levels in MCI and AD patients}

\section{Leptin}

Serum leptin levels have been extensively associated with cognitive function and risk of Alzheimer's disease [37]. However, results from several clinical studies are somehow divergent and dependent on epidemiological factors such as age, gender, and race [38]. Recent findings have shown that, despite leptin's recognized neuroprotective abilities, in some circumstances central resistance develops with consequent leptin signaling impairment, which seems to be the case in obesity and Alzheimer's disease $[39,40]$. Regardless of the fact that we did not find any significant differences in serum and CSF leptin levels between both groups, Bonda et al. (2014) previously described higher CSF leptin in AD when compared to MCI patients [40]. This could be partially explained by the fact that, in opposition to our mild to moderate stage AD cohort, the subjects included in the later study were in the latest pathological stage of disease and a six-fold increase in CSF leptin of Braak VI patients compared to Braak 0-V is described.

\section{Adiponectin}

On the other hand, few clinical studies have inconsistently reported an association between adiponectin and Alzheimer's disease (for review, see [11]) Waragai et al. (2016) recently described higher levels of adiponectin in the serum of MCI and $\mathrm{AD}$ patients when compared to controls, but no differences between the two former groups [41]. They have also shown significantly lower CSF adiponectin levels in $\mathrm{AD}$ patients compared to $\mathrm{MCI}$ and controls, although evident scattered results in the $\mathrm{AD}$ group suggest that CSF adiponectin levels may vary according to the severity or pathological stage of the disease. Overall, our results are in line with the most recent reports since serum adiponectin was $33 \%$ higher in $\mathrm{AD}$ when compared to MCI patients, albeit no differences were observed in the CSF. A possible explanation for this finding is the exclusion of severe cases in our AD cohort, which raises the possibility that higher peripheral secretion of adiponectin can maintain, for an undetermined period of time, normal CSF levels in the initial stages of the disease. Together, these results suggest that a rise in adiponectinemia may reflect an effort to compensate central defective signaling, similarly to what happens with leptin. Furthermore, we found that a cut-off of $10.85 \mu \mathrm{g} / \mathrm{ml}$ in adiponectinemia predicted the diagnosis of $\mathrm{AD}$ (versus MCI diagnosis) with $87 \%$ specificity though with only $44 \%$ sensitivity. Although this distinction does not entail considerable practical importance once $\mathrm{MCI}$ versus $\mathrm{AD}$ diagnosis rests mostly on clinical aspects, serum adiponectin could be explored as a staging biomarker, i.e., it could reflect disease progression. On the other hand, it would also be interesting to evaluate its usefulness in the differential diagnosis with other forms of dementia. Until now, the work of Bossolasco et al. (2017) was the only to show higher serum adiponectin in a non-AD type of dementia (frontotemporal dementia versus controls) [42]. Although these results counteract the usefulness of adiponectinemia as marker of $\mathrm{AD}$, very few patients have been enrolled in this study (7 FTD; 9 AD; 36 controls).

\section{Adipokines and Alzheimer's disease biomarkers}

\section{Leptin}

In this study, we report a moderate negative correlation between CSF leptin and t-tau in AD patients, reinforcing the involvement of this adipokine in tau metabolism. Noteworthy, these results oppose to those presented by Maioli et al. (2015) [43]. Although 


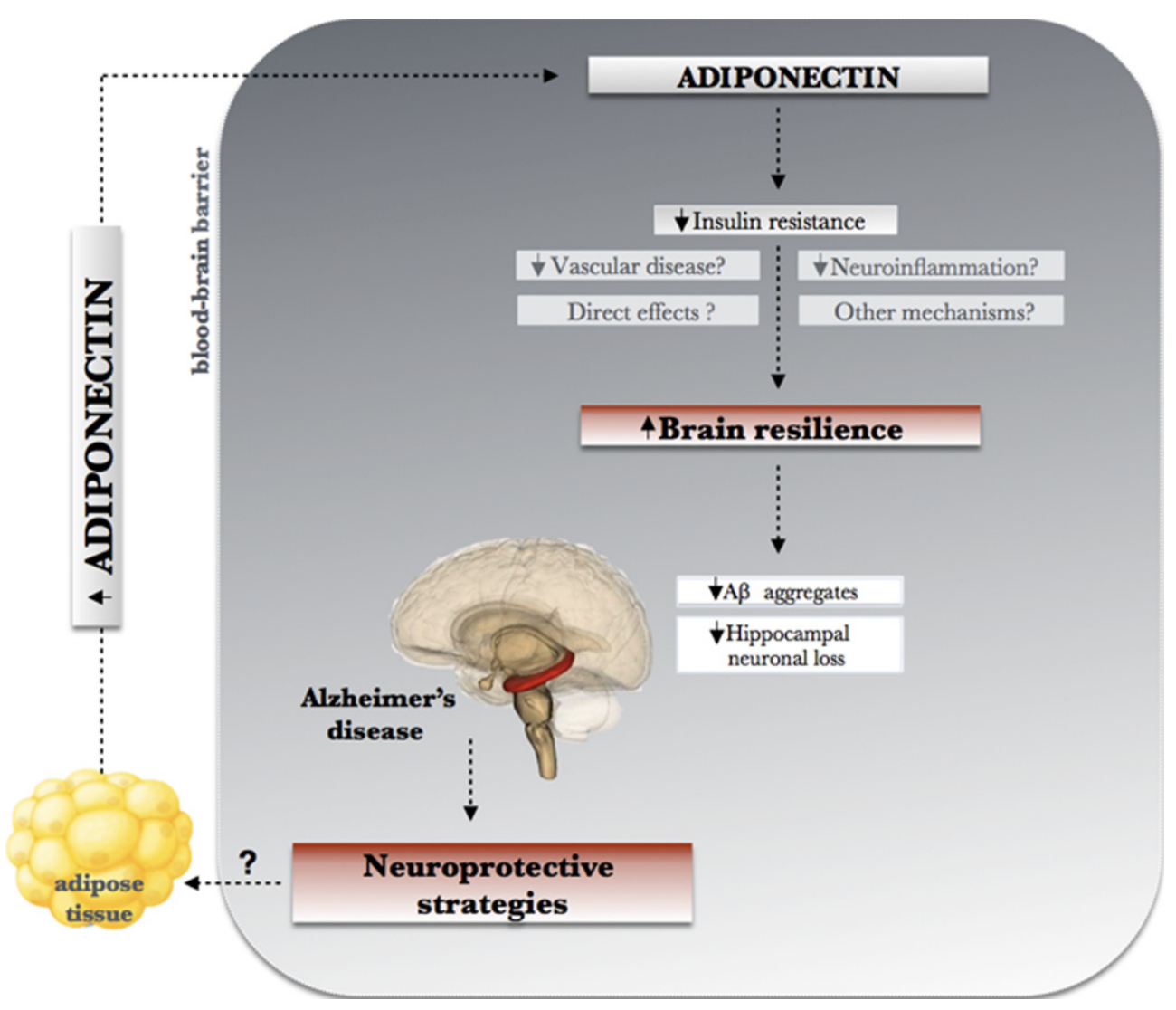

Fig. 3. Hypothetical crosstalk between Alzheimer's disease and adiponectin physiology.

it is recognized that leptin is capable of reducing tau phosphorylation [44], no correlation was observed between leptin levels and p-tau.

In contrast to previous reports [45, 46], we observed no association between serum or CSF leptin levels and MTL volumetric measures.

\section{Adiponectin}

In accordance to other studies [41, 47], our results showed no correlation between serum levels of adiponectin and Alzheimer's disease classical CSF biomarkers, and contrary to our expectations, CSF levels of adiponectin were also unrelated to $A \beta_{42}$, $\mathrm{t}$-tau, and p-tau concentrations in MCI and $\mathrm{AD}$ patients. Notably, when we separately studied female patients, we observed a strong positive correlation between CSF adiponectin and $A \beta_{42}$ suggesting that higher CSF levels of this adipokine are associated with less soluble $A \beta_{42}$, and eventually less aggregates. Our results come into line with the study conducted by Waragai et al. (2016), the first to demonstrate a correlation between CSF adiponectin and $A \beta_{42}$ as well as with p-tau in a heterogeneous cohort (controls, MCI and AD; $n=189$ ) [41]. However, they have not performed any subgroup analysis based on diagnosis, biomarker profile, or gender, and no adjustments for possible confounders such as age, gender, BMI, or APOE genotype. The co-localization of adiponectin with neurofibrillary tangles in the Alzheimer's disease brain is also interestingly described by the same authors, suggesting possible direct effects of this adipokine on pathological hallmarks of the disease.

Regarding imaging biomarkers, we found no robust correlation between CSF adiponectin concentration and MTL volumetry in MCI or AD patients neither in the female sub-group. To our knowledge, Waragai et al. (2016) was the only to report an inverse correlation between CSF adiponectin and hippocampal volume $(r=-0.291, p<0.001)$ in a combined pool of patients [41], whereas the Mayo Clinic Study of Aging published opposing data [48].

Furthermore, CSF leptin-to-adiponectin ratio did not represent an added value in the correlation study 
with Alzheimer's disease biomarkers compared to individual adipokine concentrations.

Finally, some limitations can be pointed to this study and include: 1) sample size which may be insufficient to establish epidemiological associations; 2) the absence of a pre-symptomatic group; and 3) technical impossibility to distinguish adiponectin isoforms.

\section{Conclusions}

The present study demonstrates that patients in the mild to moderate dementia phases of Alzheimer's disease present higher levels of serum adiponectin when compared to patients in its prodromal phase (MCI). Thus, adiponectin should be studied in larger cohorts to determine its usefulness as blood-based biomarker of disease progression. This study further demonstrates that higher CSF levels of this adipokine are associated with higher CSF concentration of soluble $A \beta_{42}$ and better cognition in female patients. It highlights the gender specific role that adiponectin may assume in the crosstalk between the brain and adipose tissue. In fact, it was already recognized that a gynoidlike distribution of fat (more prone to be associated with higher adiponectin levels) is associated with better cognitive performance, particularly in older women [49]. In our perspective, these findings indicate that clinical and basic research on Alzheimer's disease should always include gender specific outcomes. Furthermore, gender differences in adipose tissue's physiology and its complex CNS interactions should be included in the list of potential contributors to the female-biased AD incidence.

Although longitudinal and larger studies are required, these findings suggest that an elevation in serum adiponectin in $\mathrm{AD}$ patients may represent a homeostatic response to counterbalance neurodegeneration, taking into consideration its insulin-sensitizing, anti-inflammatory and antiapoptotic effects (Fig. 3). Furthermore, these findings also suggest that adiponectin might be specifically assigned to neuroprotective functions in women, indicating that research on Alzheimer's disease pathophysiology should always include gender specific outcomes. Ultimately, is important to outline that even with the approval of new drugs, effective and aggressive strategies for the prevention of Alzheimer's disease are imperative and a thorough understanding of its modifiable risk factors, including obesity, may have an important impact on reducing the burden of this devastating disease.

\section{ACKNOWLEDGMENTS}

We thank Rui Pascoal for his contribution in sample preparation and storage and Alice Almeida for her contribution to sample collection.

This study was supported by the Faculty of Medicine, University of Coimbra and by Portuguese Foundation for Science and Technology (project UID/NEU/04539/2013). T.R. and P.M. are supported by a $\mathrm{PhD}(\mathrm{SFRH} / \mathrm{BD} / 101172 / 2014)$ and a postdoctoral grant (SFRH/BPD/104881/2014).

Authors' disclosures available online (https:// www.j-alz.com/manuscript-disclosures/18-0669r2).

\section{SUPPLEMENTARY MATERIAL}

The supplementary material is available in the electronic version of this article: http://dx.doi.org/ 10.3233/JAD-180669 .

\section{REFERENCES}

[1] Emmerzaal TL, Kiliaan AJ, Gustafson D. 2003-2013: A decade of body mass index, Alzheimer's disease, and dementia (2015) J Alzheimers Dis 43, 739-755.

[2] Nelson AR, Sweeney MD, Sagare AP, Zlokovic BV (2016) Neurovascular dysfunction and neurodegeneration in dementia and Alzheimer's disease. Biochim Biophys Acta 1862, 887-900.

[3] Whitmer RA, Gustafson D, Barrett-Connor E, Haan MN, Gunderson EP, Yaffe K (2008) Central obesity and increased risk of dementia more than three decades later. Neurology 71, 1057-1064.

[4] Letra L, Santana I, Seiça R (2014) Obesity as a risk factor for Alzheimer's disease: The role of adipocytokines. Metab Brain Dis 29, 563-568.

[5] Galic S, Oakhill JS, Steinberg GR (2010) Adipose tissue as an endocrine organ. Mol Cell Endocrinol 316, 129-139.

[6] Harwood HJ Jr (2012) The adipocyte as an endocrine organ in the regulation of metabolic homeostasis. Neuropharmacology 63, 57-75.

[7] Stern JH, Rutkowski JM, Scherer PE (2016) Adiponectin, leptin, and fatty acids in the maintenance of metabolic homeostasis through adipose tissue crosstalk. Cell Metab 23, 770-874.

[8] Blüher M (2012) Clinical relevance of adipokines. Diabetes Metab J 36, 317-327.

[9] Scherer PE (2016) The multifaceted roles of adipose tissue-therapeutic targets for diabetes and beyond: The 2015 Banting Lecture. Diabetes 65, 1452-1461.

[10] McGuire MJ, Ishii M (2016) Leptin dysfunction and Alzheimer's disease: Evidence from cellular, animal, and human studies. Cell Mol Neurobiol 36, 203-217.

[11] Letra L, Rodrigues T, Matafome P, Santana I, Seiça R (2017) Adiponectin and sporadic Alzheimer's disease: Clinical and molecular links. Front Neuroendocrinol, doi: 10.1016/j.yfrne.2017.10.002 
[12] Zhang D, Wang X, Lu XY (2016) Adiponectin exerts neurotrophic effects on dendritic arborization, spinogenesis, and neurogenesis of the dentate gyrus of male mice. Endocrinology 157, 2853-2869.

[13] Satoh N, Naruse M, Usui T, Tagami T, Suganami T, Yamada K, Kuzuya H, Shimatsu A, Ogawa Y (2004) Leptin-toadiponectin ratio as a potential atherogenic index in obese type 2 diabetic patients. Diabetes Care 27, 2488-2490.

[14] Bravo C, Cataldo LR, Galgani J, Parada J, Santos JL (2017) Leptin/adiponectin ratios using either total or highmolecular-weight adiponectin as biomarkers of systemic insulin sensitivity in normoglycemic women. J Diabetes Res 2017, 9031079.

[15] World Health Organization (WHO) (2008) Waist circumference and waist-hip ratio: Report of a WHO expert consultation. Geneva.

[16] American Psychiatric Association (2013) Diagnostic and statistical manual of mental disorders (5thed.). Washington, DC.

[17] McKhann G, Drachman D, Folstein M, Katzman R, Price D, Stadlan EM (1984) Clinical diagnosis of Alzheimer's disease: Report of the NINCDS-ADRDA Work Group under the auspices of Department of Health and Human Services Task Force on Alzheimer's Disease. Neurology 34, 939-944.

[18] McKhann GM, Knopman DS, Chertkow H, Hyman BT, Jack CR Jr, Kawas CH, Klunk WE, Koroshetz WJ, Manly JJ, Mayeux R, Mohs RC, Morris JC, Rossor MN, Scheltens P, Carrillo MC, Thies B, Weintraub S, Phelps $\mathrm{CH}$ (2011) The diagnosis of dementia due to Alzheimer's disease: Recommendations from the National Institute on Aging-Alzheimer's Association workgroups on diagnostic guidelines for Alzheimer's disease. Alzheimers Dement 7, 263-269.

[19] Petersen RC, Smith GE, Waring SC, Ivnik RJ, Tangalos EG, Kokmen E (1999) Mild Cognitive Impairment: Clinical characterization and outcome. Arch Neurol 56, 303-308.

[20] Morris JC (1993) The Clinical Dementia Rating (CDR): Current version and scoring rules. Neurology 43, 24122414.

[21] Garrett C, Santos F, Tracana I, Barreto J, Sobral M, Fonseca R (2008) Avaliação clínica da demência - Tradução em português. Escalas e Testes na Demência. Grupo de Estudos de Envelhecimento Cerebral e Demência, Lisboa, pp. 17-32.

[22] Folstein MF, Folstein SE, McHugh PR (1975) "Mini-mental state". A practical method for grading the cognitive state of patients for the clinician. J Psychiatr Res 12, 189-198.

[23] Guerreiro M, Silva AP, Botelho MA (2003) Avaliação breve do estado mental: Escalas e testes na demência. Grupo de Estudos de Envelhecimento Cerebral e Demência, Lisboa, pp. 27-32.

[24] Nasreddine ZS, Phillips NA, Bédirian V, Charbonneau $\mathrm{S}$, Whitehead V, Collin I, Cummings JL, Chertkow H (2005) The Montreal Cognitive Assessment, MoCA: A brief screening tool for mild cognitive impairment. J Am Geriatr Soc 53, 695-699.

[25] Freitas S, Simões M, Martins C, Vilar M, Santana I (2010) Estudos de adaptação do Montreal Cognitive Assessment (MoCA) para a população portuguesa. Avaliação Psicol 9, 345-357.

[26] Mohs RC, Rosen WG, Davis KL (1983) The Alzheimer's disease assessment scale: An instrument for assessing treatment efficacy. Psychopharmacol Bull 19, 448-450.

[27] Guerreiro M, Fonseca S, Barreto J, Garcia C (2008) Escala de Avaliação da Doença de Alzheimer-EADA: Alzheimer Disease Assessment Scale ADAS. Escalas e Testes na
Demência ( $2 a$ ed). Grupo de Estudos de Envelhecimento Cerebral e Demência, Lisboa, pp. 41-68.

[28] Yesavage JA, Brink TL, Rose TL, Lum O, Huang V, Adey M, Leirer VO (1983) Development and validation of a geriatric depression screening scale: A preliminary report. J Psychiatr Res 17, 37-49.

[29] Barreto J, Leuschner A, Santos F, Sobral M (2003) Escala de depressão geriátrica,Tradução portuguesa da Geriatric Depression Inventory. Escalas e Testes na Demência (2aed). Grupo de Estudos de Envelhecimento Cerebral e Demência, Lisboa, pp. 65-67.

[30] Friedewald WT, Levy RI, Fredrickson DS (1972) Estimation of the concentration of low-density lipoprotein cholesterol in plasma, without use of the preparative ultracentrifuge. Clin Chem 18, 499-502.

[31] del Campo M, Mollenhauer B, Bertolotto A, Engelborghs S, Hampel H, Simonsen AH, Kapaki E, Kruse N, Le Bastard N, Lehmann S, Molinuevo JL, Parnetti L, Perret-Liaudet A, Sáez-Valero J, Saka E, Urbani A, Vanmechelen E, Verbeek M, Visser PJ, Teunissen C (2012) Recommendations to standardize preanalytical confounding factors in Alzheimer's and Parkinson's disease cerebrospinal fluid biomarkers: An update. Biomark Med 6, 419-430.

[32] Baldeiras IE, Ribeiro MH, Pacheco P, Machado A, Santana I, Cunha L, Oliveira CR (2009) Diagnostic value of CSF protein profile in a Portuguese population of SCJD patients. J Neurol 256, 1540-1550.

[33] Jack CR Jr, Bernstein MA, Fox NC, Thompson P, Alexander G, Harvey D, Borowski B, Britson PJ, L Whitwell J, Ward C, Dale AM, Felmlee JP, Gunter JL, Hill DL, Killiany R, Schuff N, Fox-Bosetti S, Lin C, Studholme C, DeCarli CS, Krueger G, Ward HA, Metzger GJ, Scott KT, Mallozzi R, Blezek D, Levy J, Debbins JP, Fleisher AS, Albert M, Green R, Bartzokis G, Glover G, Mugler J, Weiner M (2008) The Alzheimer's Disease Neuroimaging Initiative (ADNI): MRI methods. J Magn Reson Imaging 27, 685-691.

[34] Dale AM, Fischl B, Sereno MI (1999) Cortical surfacebased analysis. I. Segmentation and surface reconstruction. Neuroimage 9, 179-194.

[35] Fischl B, Sereno MI, Dale AM (1999) Cortical surfacebased analysis. II: Inflation, flattening, and a surface-based coordinate system. Neuroimage 9, 195-207.

[36] Mazure CM, Swendsen J (2016) Sex differences in Alzheimer's disease and other dementias. Lancet Neurol 15, 451-452.

[37] Magalhães C, Carvalho M, Sousa L, Caramelli P, Gomes K (2015) Leptin in Alzheimer' s disease. Clin Chim Acta 450, 162-168.

[38] Oania R, McEvoy LK (2015) Plasma leptin levels are not predictive of dementia in patients with mild cognitive impairment. Age Ageing 44, 53-58.

[39] Sáinz N, Barrenetxe J, Moreno-Aliaga MJ, Martínez JA (2015) Leptin resistance and diet-induced obesity: Central and peripheral actions of leptin. Metabolism 64, 35-46.

[40] Bonda DJ, Stone JG, Torres SL, Siedlak SL, Perry G, Kryscio R, Jicha G, Casadesus G, Smith MA, Zhu X, Lee HG (2014) Dysregulation of leptin signaling in Alzheimer disease: Evidence for neuronal leptin resistance. $\mathrm{J} \mathrm{Neu}$ rochem 128, 162-172.

[41] Waragai M, Adame A, Trinh I, Sekiyama K, Takamatsu Y, Une K, Masliah E, Hashimoto M (2016) Possible involvement of adiponectin, the anti-diabetes molecule, in the pathogenesis of Alzheimer's disease. J Alzheimers Dis 52, 1453-1459. 
[42] Bossolasco P, Cancello R, Doretti A, Morelli C, Silani V, Cova L (2017) Adiponectin levels in the serum and cerebrospinal fluid of amyotrophic lateral sclerosis patients: Possible influence on neuroinflammation? J Neuroinflammation 14, 85.

[43] Maioli S, Lodeiro M, Merino-Serrais P, Falahati F, Khan W, Puerta E, Codita A, Rimondini R, Ramirez MJ, Simmons A, Gil-Bea F, Westman E, Cedazo-Minguez A; Alzheimer's Disease Neuroimaging Initiative (2015) Alterations in brain leptin signalling in spite of unchanged CSF leptin levels in Alzheimer's disease. Aging Cell 14, 122-129.

[44] Greco SJ, Sarkar S, Johnston JM, Zhu X, Su B, Casadesus G, Ashford JW, Smith MA, Tezapsidis N (2008) Leptin reduces Alzheimer's disease-related tau phosphorylation in neuronal cells. Biochem Biophys Res Commun 376, 536541.

[45] Lieb W, Beiser AS, Vasan RS, Tan ZS, Au R, Harris TB, Roubenoff R, Auerbach S, DeCarli C, Wolf PA, Seshadri $S$ (2009) Association of plasma leptin levels with incident Alzheimer disease and MRI measures of brain aging. JAMA 302, 2565-2572.
[46] Rajagopalan P, Toga AW, Jack CR, Weiner MW, Thompson PM; Alzheimer's Disease Neuroimaging Initiative (2013) Fat-mass-related hormone, plasma leptin, predicts brain volumes in the elderly. Neuroreport 24, 58-62.

[47] Diehl-Wiesenecker E, von Armin CA, Dupuis L, Müller HP, Ludolph AC, Kassubek J (2015) Adipose tissue distribution in patients with Alzheimer's disease: A whole body MRI case-control study. J Alzheimers Dis $\mathbf{4 8}$, 825-832.

[48] Wennberg AM, Gustafson D, Hagen CE, Roberts RO, Knopman D, Jack C, Petersen RC, Mielke MM (2016) Serum adiponectin levels, neuroimaging, and cognition in the Mayo Clinic Study of Aging. J Alzheimers Dis 53, 573581.

[49] Forte R, Pesce C, de Vito G, Boreham CAG (2016) The body fat-cognition relationship in healthy older individuals: Does gynoid vs android distribution matter? J Nutr Health Aging 21, 284-292. 